\title{
Dewetting at soft viscoelastic interfaces
}

\author{
G. Carbone ${ }^{\mathrm{a})}$ \\ DIMeG Politecnico di Bari, V.le Japigia 182, 70126 Bari, Italy and IFF Forschungszentrum Jülich, \\ 52425 Jülich, Germany \\ B. N. J. Persson ${ }^{\text {b) }}$ \\ IFF Forschungszentrum Jülich, 52425 Jülich, Germany
}

(Received 15 October 2003; accepted 12 May 2004)

\begin{abstract}
The dewetting transition of thin liquid films $(\sim 100 \mathrm{~nm})$ at soft viscoelastic interfaces is analyzed theoretically. It is shown that viscoelastic losses in the soft material can drastically increase the time to complete the dewetting. Thus, the influence of the thinning of the liquid film, due to the hydrodynamic drainage caused by the external applied pressure, has to be considered too. The squeezing pressure coupled with the hydrodynamic drainage may slow down the dewetting to almost zero growth rate of the dry zone; in this case a trapped rim of fluid should be observed. (c) 2004 American Institute of Physics. [DOI: 10.1063/1.1768156]
\end{abstract}

\section{INTRODUCTION}

Dewetting of a liquid film interposed between a flat rigid surface (e.g., a glass surface) and a soft rubberlike material has been studied for many years because of its great scientific interest and practical importance. In some cases it is necessary to have a fast dewetting to assure direct contact between the rubber and the rigid substrate (e.g., friction of tires on a wet road $\left.{ }^{1,2}\right)$. In other situations the rupture of the liquid film should be prevented in order to avoid damages as, for instance, in the case of silicone contact lenses where the rupture of the lacrimal film has been observed: the lens adheres strongly to the surface of cornea thus causing severe damages during its removal. Dewetting is also of great importance in biological applications involving cell adhesion to a substrate. ${ }^{3,4}$

Two different limiting scenarios have been observed during the squeezing out of a liquid interposed between two smooth solid surfaces. For hard solid walls ${ }^{5-10}$ (e.g., metal walls) separated by less than $10 \mathrm{ML}$ (monolayer) of the liquid, the squeezing process occurs in a quantized manner: a single monolayer is squeezed out at each step (see Fig. 1). The layering transition usually nucleates in the central region of the contact area, where the pressure ( $\sim 1 \mathrm{GPa})$ is the highest and proceeds with a circular shape toward the periphery. The large elastic modulus $E \sim 10^{11} \mathrm{~Pa}$ of the solid walls prevents a liquid rim to be formed at the boundary line between the $n$ and $n-1$ monolayers region (where $n$ is the number of trapped layers). On the other hand, when the solid walls are sufficiently soft, as for rubberlike materials $\left(E \sim 10^{6} \mathrm{~Pa}\right)$, a different scenario takes place: ${ }^{11-14}$ the squeeze-out process nucleates at some defects on the solid walls when the film is quite thick $(\sim 100 \mathrm{~nm})$ causing an abrupt reduction to zero thickness. The liquid expelled is collected in a thick rim of fluid surrounding the just formed dry zone (see Fig. 2). The rim can be formed because of the small energy required to

\footnotetext{
${ }^{a)}$ E-mail address: Carbone@poliba.it

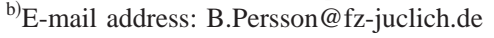

deform the soft elastic rubber wall, and the dewetting proceeds until the rim reaches the boundary of the original contact area. The commonly accepted theory ${ }^{11-14}$ assumes that the driving force for squeeze out is due to the change of the interfacial energy, and that the perpendicular pressure distribution has no influence on the squeeze out dynamics. Thus the growth rate of the dry zone is determined by a simple energy balance equation between the work of adhesion per unit time and the energy dissipated per unit time in the viscous fluid in the rim. However, this argument is only valid when the pressure over the contact is uniform and the viscoelastic losses in the rubber are negligible.

In a previous paper ${ }^{15}$ it has been shown that the contribution to the driving force coming from the spatial variation of the pressure over the contact (e.g., Hertzian pressure distribution) cannot be neglected, being in some cases much more important than the contribution of the interfacial energy. In this paper we consider rubberlike materials which exhibit strong viscoelastic behavior (i.e., large internal friction losses). In accordance with some existing works, ${ }^{16,17}$ the estimation of the viscoelastic losses in the rubber is done by considering a very simple rheological model with a unique relaxation time. Moreover, the detailed shape of the $\operatorname{rim}^{17,18}$ is not taken into account when we estimate the dissipation of energy in the rubber. Therefore we expect that the viscoelastic losses are underestimated in our model, since the influence of the singular stress behavior close to the tip of the rim is not taken into account. However, we believe that this does not change the basic physics, and the conclusions we derived in the paper. We show that the energy dissipation in the rubber, close to the moving rim, can produce a resistant force that strongly slows down the growth rate of the dry zone. Thus the time to complete the dewetting rapidly increases, and the thinning of the liquid film due to the hydrodynamic drainage can no more be neglected. This necessarily complicates the overall treatment, since both the evolution of the extension of the dry patch and that of the liquid thickness in the annular external region have to be calculated at the same time. 


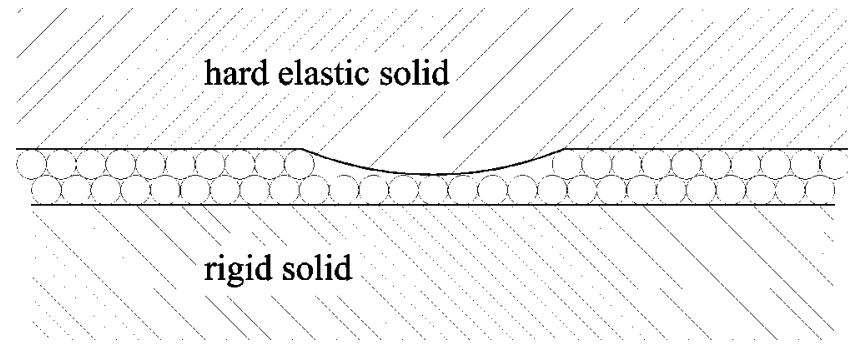

FIG. 1. The squeeze out of a fluid between a hard elastic surface and a rigid substrate. During the layering transition $n \rightarrow n-1 \quad(n=2$ in the figure) the surfaces are everywhere separated by $n$ or $n-1$ monolayers of fluid.

Notice that in this case, if the average pressure is quite high $(0.1-1 \mathrm{MPa})$, the thinning of the fluid film, due to the hydrodynamic drainage, occurs very rapidly thus producing also a strong reduction of the growth rate of the volume of the rim. This has the following two important consequences.

(1) The mean value of the pulsating frequency grows because of the smaller dimensions of the rim. Thus the resistant force due to the viscoelasticity of the rubber increases and the spreading velocity of the dry patch is reduced.

(2) The driving force due to the nonuniform pressure distribution decreases because of the smaller volume of the liquid rim, thus producing a further slowing down of the dewetting transition.

Therefore, it is expected that for a soft viscoelastic material, under sufficiently high squeezing pressures, the dewetting process may be very slow, and a quasistationary rim of trapped liquid may be formed in the contact region.

\section{DEWETTING TRANSITION}

Consider a thin liquid film interposed between a soft material, e.g., rubber, and a flat rigid surface, as, for instance, a glass surface. Suppose that the spreading parameter is negative:

$$
\Delta \gamma=\gamma_{R S}-\left(\gamma_{R L}+\gamma_{L S}\right)<0,
$$

where $\gamma_{R S}, \gamma_{R L}$, and $\gamma_{L S}$ are the rubber/solid, rubber/liquid, and liquid/solid interfacial energies. In this case the flat liquid film between the rubber and the substrate is unstable and may dewet by the nucleation and growth of a dry patch surrounded by a liquid rim collecting the expelled fluid. ${ }^{11-14}$

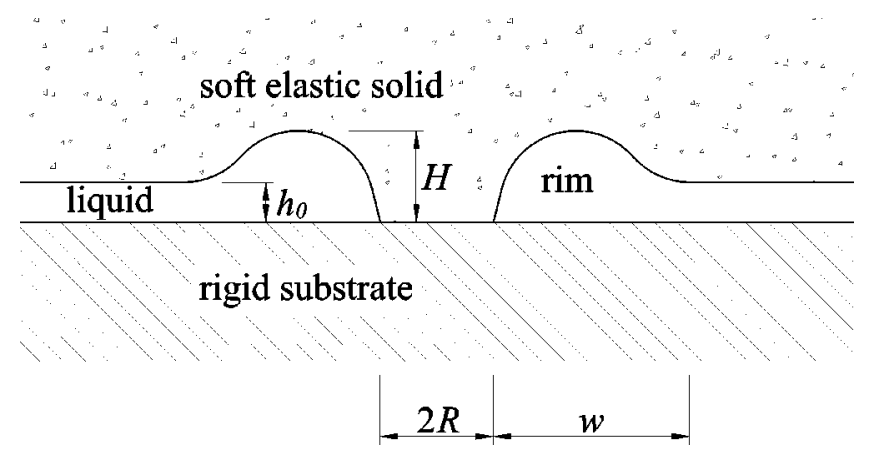

FIG. 2. The squeeze out of a nonwetting liquid between a hard rigid and a soft elastic material. The squeezed liquid collects in a rim.

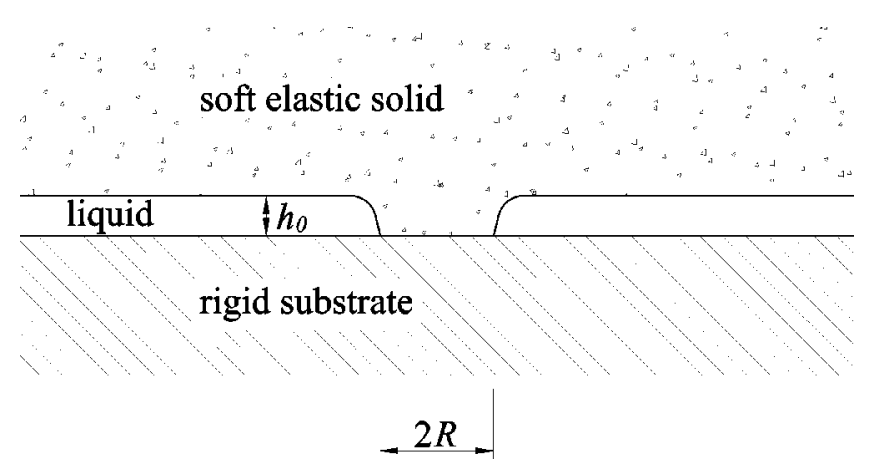

FIG. 3. The nucleation of the dewetting transition of a nonwetting liquid interposed between a soft elastic material and a hard flat substrate.

\section{A. Nucleation}

Let $h_{0}$ be the initial thickness of the fluid film and let us evaluate the energy required for nucleation, i.e., to generate a bridge of radius $R$ and thickness $h_{0}$, see Fig. 3. The stored elastic energy, neglecting factors of order unity, is $U_{E}$ $\simeq E_{0} \varepsilon^{2} V$, where $E_{0}=E(\omega=0)$ is the elastic modulus of the rubber at zero frequency, $\varepsilon \simeq h_{0} / R$ is the strain, and $V \simeq R^{3}$ is the volume of solid over which the elastic displacement extends. Therefore we can write $U_{E} \simeq E_{0}\left(h_{0} / R\right)^{2} R^{3}=E h_{0}^{2} R$. On the other hand, the gain in surface energy is $U_{A}$ $=\pi R^{2} \Delta \gamma$. Hence the total energy is

$$
U \simeq E_{0} h_{0}^{2} R+\pi R^{2} \Delta \gamma .
$$

The critical radius $R_{c}$ for nucleation can be calculated by the condition $\partial U / \partial R=0$, which gives

$$
R_{c} \simeq \frac{1}{2 \pi} \frac{h_{0}^{2}}{e},
$$

where

$$
e=\frac{|\Delta \gamma|}{E_{0}}
$$

is a characteristic length usually in the range $10-100 \mathrm{~nm}$. If the radius of the bridge is smaller than the critical radius $R_{c}$ the bridge closes back, while if the radius of the bridge is larger than the critical value $R_{c}$, the dry area will expand until complete dewetting has occurred. Observe that $R_{c}$ grows with the second power of the film thickness $h_{0}$, so that spontaneous nucleation of squeeze out can only occur for very thin film. In fact for perfectly flat surfaces the nucleation would take place when the energy barrier $\Delta U$ $\simeq(1 / 4 \pi)|\Delta \gamma| e^{2}\left(h_{0} / e\right)^{4} \quad$ equals $\quad \Delta U \simeq k_{B} T \ln \nu \tau_{N} \simeq 1 \mathrm{eV}$, where $\nu \sim 10^{16} \mathrm{~s}^{-1}$ is a very large prefactor ${ }^{15}$ and $\tau_{N} \sim 1 \mathrm{~s}$ is a macroscopic time. Therefore for rubberlike material $\left(E_{0}\right.$ $\simeq 1 \mathrm{MPa}$ and $|\Delta \gamma| \simeq 1 \mathrm{meV} / \AA^{2}$ ) the corresponding film thickness would be $h_{0} \simeq 10 \mathrm{~nm}$ and the critical radius $R_{c}$ $\simeq 2 \mathrm{~nm}$. But in practical situations the nucleation occurs for much larger film thickness $\sim 100 \mathrm{~nm}$ indicating that it is induced by defects or imperfections at the interface.

\section{B. Spreading}

Assume that the dewetting transition nucleates at some imperfections of the walls when the fluid is still quite thick 
$\sim 100 \mathrm{~nm}$. The rubber surface deforms to create a rim that collects the fluid expelled from the dry patch, see Fig. 2. The profile of the rim is quasistatic and very similar to the shape of a sessile droplet, hence the following scaling relation holds: ${ }^{11,14-19}$

$$
w=\frac{H^{2}}{e} \text {. }
$$

After nucleation the dry patch surrounded by the liquid rim expands until the dewetting is complete. The standard theory ${ }^{11-14}$ assumes that the rubber is perfectly elastic and neglects the influence of the spatial variation of pressure distribution over the contact area. The gain in surface energy is, therefore, completely dissipated in the fluid rim because of its viscous behavior. The theoretical scaling relation $R \simeq t^{3 / 4}$ fits well some classical experimental results obtained for high-viscosity liquids. In order to observe the evolution of the system, the dewetting transition was slowed down by choosing a fluid with a sufficiently high viscosity $\sim 1-20 \mathrm{~Pa}^{13}$ Moreover, the soft material was a PolyDimethylSiloxane (PDMS) rubber with negligible hysteretic properties and the squeezing pressure was limited to $\sim 0.1 \mathrm{MPa}$. In a previous paper ${ }^{15}$ it has been shown that in many practical situations the nonuniformity of the pressure distribution increases the driving force, producing thus a much faster dewetting transition. But it was also pointed out that for strongly viscoelastic materials the dewetting behavior could be very different.

In this paper we study the influence of the hysteretic properties of the rubber and the hydrodynamic drainage of the fluid on the spreading behavior of the dry zone.

The negative work per unit time done by the internal frictional stresses, neglecting the singular behavior of the stress $\sigma \sim 1 / \sqrt{r}$ near the crack tip, can be estimated as

$$
P_{R}=V \omega \varepsilon_{0}^{2} \operatorname{Im} E(\omega)=4 \pi^{2} R H^{2} \frac{\dot{R}}{w} \operatorname{Im} E(\omega) .
$$

Neglecting factors of order unity, the work per unit time done by the viscous stresses in the fluid rim $P_{F}$ can be estimated as

$$
P_{F}=-\eta\left(\frac{\dot{R}}{H}\right)^{2} \Delta V=-\eta\left(\frac{\dot{R}}{H}\right)^{2} 2 \pi R w H .
$$

Here $\varepsilon_{0}=H / w$ is the strain in the deformed rubber, $V$ $=2 \pi R w^{2}$ is the volume of the deformed rubber, $\omega$ $=2 \pi \dot{R} / w$ is the pulsating frequency and $\Delta V=2 \pi R w H$ is the volume of the rim (we are considering $w \ll R$ and $h_{0}$ $\ll H)$. In order to calculate the energy dissipated in the rubber, the frequency dependence of the complex elastic modulus $E(\omega)$ should be known. Here we use a very simple model (see Fig. 4), corresponding to the following complex elastic modulus:

$$
E(\omega)=E_{\infty} \frac{1-i \omega \tau_{R}}{1+a-i \omega \tau_{R}},
$$

where $E_{\infty}=E(\omega \rightarrow \infty),(1+a)=E_{\infty} / E_{0}$, and $\tau_{R}$ is a characteristic time constant of the soft material. This model is not a very good description of the behavior of real rubber, since

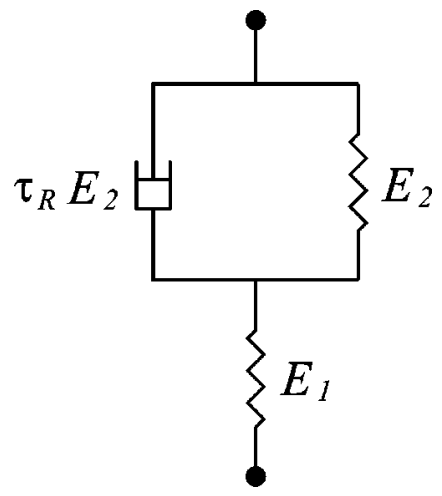

FIG. 4. The viscoelastic model used for a qualitative description of the rubber behavior $\left(E_{1}=E_{\infty}, E_{2}=E_{\infty} / a\right)$.

the transition from the rubbery to the glassy region is too abrupt as the frequency increases. However, the model gives a qualitatively correct complex modulus. Equation (7) yields

$$
\operatorname{Im} E(\omega)=-a E_{0} \frac{2 \pi \tau_{R} \dot{R} /[w(1+a)]}{1+\left\{2 \pi \tau_{R} \dot{R} /[w(1+a)]\right\}^{2}} .
$$

(Observe that the maximum value of $|\operatorname{Im} E(\omega)|=a E_{0} / 2$ is obtained for $2 \pi \tau_{R} \dot{R} /[w(1+a)]=1$ but in practical situations $2 \pi \tau_{R} \dot{R} /[w(1+a)] \ll 1$, so it is expected that a reduction of the volume of the rim, which turns into a reduction of $w$, causes the pulsating frequency $2 \pi \dot{R} / w$ to increase, thus producing a larger viscoelastic energy dissipation, see also Eq. (5).) Let us calculate the work per unit time done by the adhesive forces:

$$
P_{A}=\frac{d}{d t}\left(-\Delta \gamma \pi R^{2}\right)=-2 \pi \Delta \gamma R \dot{R}
$$

and by the pressure distribution over the whole contact area of radius $R_{0}$ :

$$
\begin{aligned}
P_{p}= & -\Delta V p^{\prime} \cdot(R) \dot{R}-\frac{d \Delta V}{d t} p(R)+2 \pi p(R) h R \dot{R} \\
& -\int_{R}^{R_{o}} 2 \pi \rho p(\rho) \dot{h} d \rho .
\end{aligned}
$$

Assuming a uniform thickness $h$, and considering a Hertzian pressure distribution, gives

$$
\begin{aligned}
P_{p}= & 3 \pi w H p_{0}\left(R^{2} / R_{0}^{2}\right)\left(1-R^{2} / R_{0}^{2}\right)^{-1 / 2} \dot{R} \\
& -2 \pi p(R) d(R w H) / d t+2 \pi p(R) h R \dot{R} \\
& -\pi p_{0} R_{0}^{2}\left(1-R^{2} / R_{0}^{2}\right)^{3 / 2} \dot{h} .
\end{aligned}
$$

The negative work per unit time done by the viscous stresses of the liquid flowing in the external ring shaped wet region $R<\rho \leqslant R_{0}$ is, neglecting factors of order unity,

$$
P_{H}=-\int_{R}^{R_{0}} \eta\left(\frac{\dot{\rho}}{h}\right)^{2} 2 \pi h \rho d \rho=-\frac{2 \pi}{h} \eta \int_{R}^{R_{0}} \rho \dot{\rho}^{2} d \rho .
$$

We also need the continuity equation: 


$$
2 \pi \dot{R} R h-\frac{d \Delta V}{d t}-\pi\left(\rho^{2}-R^{2}\right) \dot{h}-2 \pi \rho h \dot{\rho}=0 .
$$

Equation (13) is valid for all values of the radial coordinate $\rho$, thus by choosing $\rho=R+w=R^{+}$in Eq. (13) we get the special case

$$
h R\left(\dot{R}-V_{R}\right)=\frac{d}{d t}(R w H) .
$$

The radial velocity of the fluid $V_{R}$ at $\rho=R+w=R^{+}$is such that $V_{R} / \dot{R} \ll 1$. Thus, Eq. (14) gives

$$
h R \dot{R} \simeq \frac{d}{d t}(R w H) .
$$

Substituting Eq. (15) in Eq. (11) gives

$P_{p}=\frac{3}{2} p_{0} \Delta V \frac{R}{R_{0}^{2}} \dot{R}\left[1-\left(\frac{R}{R_{0}}\right)^{2}\right]^{-1 / 2}-\pi p_{0} R_{0}^{2} \dot{h}\left[1-\left(\frac{R}{R_{0}}\right)^{2}\right]^{3 / 2}$.

and substituting Eq. (15) in Eq. (13) gives

$$
\dot{\rho}=-\frac{1}{2} \frac{\rho^{2}-R^{2}}{\rho} \frac{\dot{h}}{h} .
$$

Equation (17) is basically the same as Eq. (13), where we have used Eq. (14) with the condition $V_{R} / \dot{R} \ll 1$ to eliminate the time derivative of $R$. Thus, by means of Eq. (17), it is possible to calculate the integral in Eq. (12) to obtain

$$
\begin{aligned}
P_{H}= & -\frac{\pi}{8} \frac{\eta R_{0}^{4}}{h^{3}} \dot{h}^{2}\left[1+3\left(\frac{R}{R_{0}}\right)^{4}-4\left(\frac{R}{R_{0}}\right)^{2}\right. \\
& \left.-4\left(\frac{R}{R_{0}}\right)^{4} \ln \frac{R}{R_{0}}\right] .
\end{aligned}
$$

Due to the hydrodynamic drainage effect, the film thickness $h$ in the external annular region is not constant, and its time evolution has to be calculated together with that of the extension $R$ of the dry patch. To solve this complicated problem we use the principle of virtual work which gives

$$
\delta L_{A}+\delta L_{R}+\delta L_{F}+\delta L_{p}+\delta L_{H}=0,
$$

where

$$
\begin{aligned}
\delta L_{A}= & -2 \pi \Delta \gamma R \delta R, \\
\delta L_{R}= & 4 \pi^{2} \frac{R}{w} H^{2} \operatorname{Im} E(\omega) \delta R, \\
\delta L_{F}= & -\eta 2 \pi R \frac{w}{H} \dot{R} \delta R, \\
\delta L_{p}= & 3 \pi w H p_{0} \frac{R^{2}}{R_{0}^{2}}\left(1-\frac{R^{2}}{R_{0}^{2}}\right)^{-1 / 2} \delta R \\
& -\pi p_{0} R_{0}^{2}\left(1-\frac{R^{2}}{R_{0}^{2}}\right)^{3 / 2} \delta h, \\
\delta L_{H}= & -\frac{\pi}{8} \frac{\eta R_{0}^{4}}{h^{3}}\left(1+3 \frac{R^{4}}{R_{0}^{4}}-4 \frac{R^{2}}{R_{0}^{2}}-4 \frac{R^{4}}{R_{0}^{4}} \ln \frac{R}{R_{0}}\right) \dot{h} \delta h .
\end{aligned}
$$

The variations $\delta R$ and $\delta h$ are independent, i.e., not correlated, thus Eq. (19) and Eqs. (20)-(24) allow us to derive the following two independent equations:

$$
\begin{aligned}
&|\Delta \gamma|+2 \pi \frac{H^{2}}{w} \operatorname{Im} E(\omega)-\eta \frac{w}{H} \dot{R} \\
&+\frac{3}{2} w H p_{0} \frac{R}{R_{0}^{2}}\left[1-\left(\frac{R}{R_{0}}\right)^{2}\right]^{-1 / 2}=0, \\
& p_{0}\left[1-\left(\frac{R}{R_{0}}\right)^{2}\right]^{3 / 2}+\frac{1}{8} \frac{\eta R_{0}^{2}}{h^{3}}\left(1+3 \frac{R^{4}}{R_{0}^{4}}-4 \frac{R^{2}}{R_{0}^{2}}\right. \\
&\left.-4 \frac{R^{4}}{R_{0}^{4}} \ln \frac{R}{R_{0}}\right) \dot{h}=0 .
\end{aligned}
$$

Let us define

$r=R / R_{0}, \quad \widetilde{H}=\left[2 H^{3} /\left(e h_{0} R_{0}\right)\right]^{1 / 3}, \quad \widetilde{h}=h / h_{0}, \quad \tilde{t}=t / \tau$,

$\kappa=\frac{3}{4} h_{0} p_{0} /|\Delta \gamma|, \quad \chi=\left[h_{0}^{2} /\left(2 e R_{0}\right)\right]^{1 / 3}$,

$\zeta=[1 /(2 \pi)](1+a) \tau / \tau_{R}$,

where the characteristic time $\tau$ is

$$
\tau=\frac{3}{4} \frac{\eta}{|\Delta \gamma|}\left(\frac{h_{0} R_{0}^{4}}{2 e^{2}}\right)^{1 / 3} .
$$

Using Eq. (4), Eqs. (25) and (26) become

$$
\begin{aligned}
1+\kappa & \widetilde{H}^{3} r\left(1-r^{2}\right)^{-1 / 2}-2 \pi a(\zeta \chi) \widetilde{H}^{2} \frac{r^{\prime}(\widetilde{t})}{(\zeta \chi)^{2} \widetilde{H}^{4}+r^{\prime}(\widetilde{t})^{2}} \\
& -\frac{4}{3} \widetilde{H} r^{\prime}(\widetilde{t})=0,
\end{aligned}
$$

$\widetilde{h}^{\prime}(\widetilde{t})=-8 \kappa \chi^{2} \frac{\left(1-r^{2}\right)^{3 / 2}}{1+3 r^{4}-4 r^{2}-4 r^{4} \ln r} \widetilde{h}^{3}$.

Equation (29) is a third-order algebraic equation of the unknown $r^{\prime}(\tilde{t})$ that can be solved symbolically and admits only one real solution (the other two being complex conjugate) of the form

$$
r^{\prime}(\widetilde{t})=f(r, \widetilde{H}, \kappa, \zeta \chi) .
$$

Notice that $f(r, \widetilde{H}, \kappa, \zeta \chi)$ does not depend separately on $\zeta$ and $\chi$, but only on their product. Finally Eqs. (15), (4), and (31) give

$$
\widetilde{H}^{\prime}(\widetilde{t})=\frac{1}{3}\left(2 \frac{\widetilde{h}}{\widetilde{H}^{2}}-\frac{\widetilde{H}}{r}\right) f(r, \widetilde{H}, \kappa, \zeta \chi),
$$

where the dimensionless quantities have been defined in Eq. (27).

Hence the governing set of equations is

$$
\begin{aligned}
r^{\prime}(\tilde{t}) & =f(r, \widetilde{H}, \kappa, \zeta, \chi), \\
\tilde{h}^{\prime}(\tilde{t}) & =-8 \chi^{2} \kappa \frac{\left(1-r^{2}\right)^{3 / 2}}{1+3 r^{4}-4 r^{2}-4 r^{4} \ln r} \widetilde{h}^{3},
\end{aligned}
$$




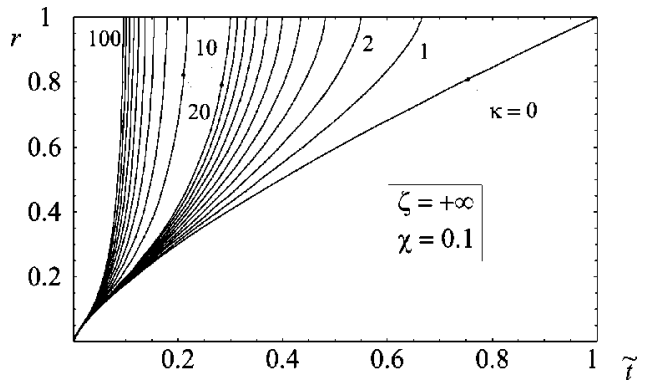

FIG. 5. The dimensionless radius $r$ of the dry patch as a function of the dimensionless squeeze-out time $\tilde{t}$. Results are shown for a perfectly elastic soft material, i.e., $\zeta \rightarrow+\infty$, and for several values of the pressure para meter $\kappa$.

$$
\widetilde{H}^{\prime}(\widetilde{t})=\frac{1}{3}\left(2 \frac{\widetilde{h}}{\widetilde{H}^{2}}-\frac{\widetilde{H}}{r}\right) f(r, \widetilde{H}, \kappa, \zeta \kappa) .
$$

This set of ordinary differential equations can be easily solved by means of a numerical procedure once the initial set of values have been assigned. It is clear from the above discussion that the dewetting process can occur only when the radius $R(t=0)$ of the dry zone is larger than the critical value $R_{c}$ given by Eq. (2). Hence a possible choice for the set of initial values is

$$
\begin{aligned}
& r(0)=R_{c} / R_{0}, \\
& \widetilde{h}(0)=1, \\
& \widetilde{H}(0)=\left(R_{c} / R_{0}\right)^{1 / 3} .
\end{aligned}
$$

Equation (38) has been obtained by considering that when the nucleation takes place this occurs abruptly so that there is no time for the fluid film to reduce its thickness below the initial value $h_{0}$. Hence Eq. (35) with $\widetilde{h}=1$ gives $\widetilde{H}(0)$ $=r(0)^{1 / 3}$ that is the same as Eq. (38).

\section{RESULTS AND DISCUSSION}

For rubberlike materials the characteristic length $e$ $=|\Delta \gamma| / E_{0}$ can be calculated considering that $E_{0} \simeq 1 \mathrm{MPa}$ and $|\Delta \gamma| \simeq 10^{-2} \mathrm{~J} / \mathrm{m}^{2}$, which gives $e \simeq 10 \mathrm{~nm}$. Moreover since $h_{0} \simeq 100 \mathrm{~nm}$, taking $2 R_{0} \simeq 1 \mathrm{~mm}$ it follows that $\chi$ $\simeq 0.1$. This value of the dimensionless parameter $\chi$ will be kept constant in the following calculations. We also use $a$ $\simeq 1000$, as is typical for rubber. In Fig. 5 we show the dimensionless radius $r$ of the dry patch versus the dimensionless time $\tilde{t}$ for several value of the dimensionless pressure parameter $\kappa$. Results are shown for a perfectly elastic material $(\zeta \rightarrow+\infty)$. We point out that the curves in Fig. 5 are very close to those obtained by one of us in a previous paper ${ }^{15}$ and confirm that, at least when the rubber behaves as an almost perfectly elastic material, the contribution to the driving force from the spatial variation of the pressure distribution considerably speeds up the dewetting transition. However, some differences appear due to the influence of the hydrodynamic flow that produces an increasingly large reduction of the film thickness as the average pressure increases. Figure 6 shows that for large values of the pressure parameter $\kappa$ the

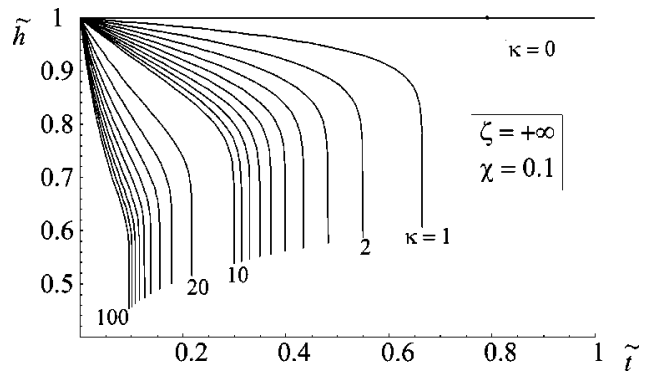

FIG. 6. The dimensionless thickness $\tilde{h}$ of the fluid film as a function of the dimensionless squeeze-out time $\tilde{t}$. Results are shown for a perfectly elastic soft material, i.e., $\zeta \rightarrow+\infty$, and for several values of the pressure para meter $\kappa$.

reduction of the film thickness can be considerable, up to $\sim 50 \%$. Therefore we expect that, due to the film thickness reduction, the flow rate at which the liquid collects into the rim [see Eq. (15)] rapidly decreases as the pressure increases, thus resulting in a much slower growth of the volume $\Delta V$ $=2 \pi R H w$ of the rim. Since the contribution of the pressure distribution to the dewetting driving force is proportional both to the average pressure $p_{0}$ and to the volume of the rim $\Delta V$ [see Eq. (16)], we deduce that the speeding up of the rim due to this contribution has been overestimated in Ref. 15 . Figure 7 shows, indeed, that the percentage increment $\Delta t \%$ of the time to complete dewetting, caused by the hydrodynamic drainage of the liquid, increases with the average pressure, up to $\sim 10 \%$ for $\kappa=100$. We expect, however, that the slowing down of the dewetting transition due this effect will be amplified as the viscoelastic losses in the rubber grows.

In Fig. 8 it is shown that by increasing the hysteretic behavior of the soft material, i.e., by reducing the dimensionless parameter $\zeta$, the growth rate of the dry patch continuously decreases and the time for complete dewetting continuously increases (results are shown for zero external pressure $\kappa=0)$ [In Fig. 8 the curves are plotted for several values of the product $\zeta \chi$, because for $\kappa=0$ the evolution of the system, as given by Eqs. (33)-(35), does not depend separately on $\zeta$ and $\chi$ but only on their product.].

Let us now consider what happens when we include the viscoelastic losses, and allow the external pressure to vary. In Fig. 9 we plot the time to complete the squeeze out, $\widetilde{t}(r$ $=1)$, as a function of the parameter $\zeta$ for several values $(\kappa$

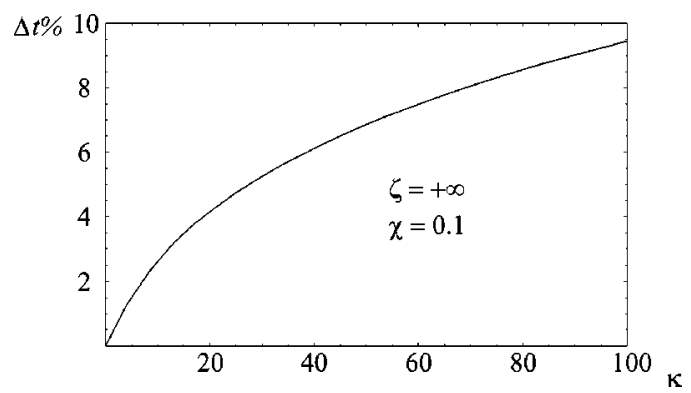

FIG. 7. The percentage change $\Delta t \%$ of the time for complete dewetting caused by the hydrodynamic drainage of the liquid as a function of the dimensionless pressure parameter $\kappa$. Results are shown considering a perfectly elastic soft material, i.e., $\zeta \rightarrow+\infty$. 


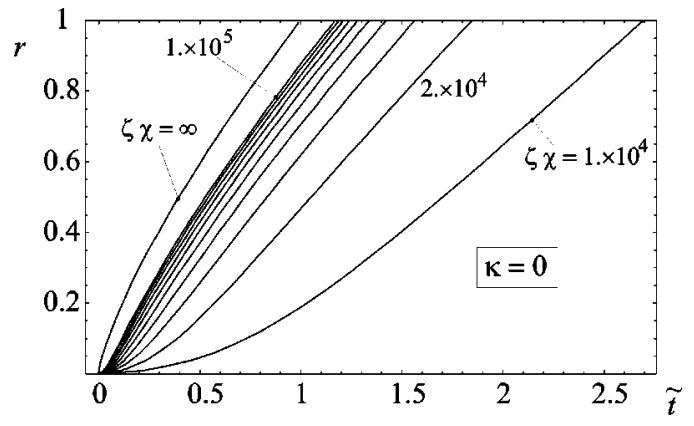

FIG. 8. The dimensionless radius $r$ as a function of the dimensionless squeeze-out time $\tilde{t}$. Results are shown for no load conditions, i.e., $\kappa=0$, and for several values of the parameter $\zeta$ : the smaller $\zeta$ the larger the viscoelastic losses in the rubber. The curves are plotted for several values of the product $\zeta \chi$, because for $\kappa=0$ the evolution of the system, as given by Eqs. (33)(35), does not depend separately on $\zeta$ and $\chi$ but only on their product.

$=0,1,10,100)$ of the dimensionless parameter $\kappa$. It is clear that for large enough viscoelastic losses (say $\zeta<10^{5}$ for $\chi$ $=0.1$ ) the time required to complete the dewetting may be three or four order of magnitude longer than for the case of purely elastic rubber. Simultaneously the thickness of the liquid, as shown in Fig. 10, rapidly decreases. It is interesting to observe (see Fig. 9) that for large enough values of $\zeta$ (say $\zeta>10^{6}$ for $\chi=0.1$ ), an increment of the average pressure $p_{0}$ ( $\kappa$ increases) produce a speeding up of the dewetting process, while for $\zeta<10^{5}$ the opposite effect is observed. This surprising result can be easily understood by observing that the term in Eq. (29) related to the viscoelastic losses,

$$
2 \pi a(\zeta \chi) \widetilde{H}^{2} \frac{r^{\prime}(\tilde{t})}{(\zeta \chi)^{2} \widetilde{H}^{4}+r^{\prime}(\widetilde{t})^{2}},
$$

grows as $\zeta$ decreases (say from $+\infty$ to $10^{3}$ ). The physical explanation is simple: the external squeezing pressure causes a thinning of the liquid film, which, in turn, decreases the growth rate of the volume $\Delta V=2 \pi R w H=2 \pi R H^{3} / e$ of the rim [see Eq. (15)]. The reduction of $\Delta V$ causes a reduction of $H$, and therefore of $w$ [see Eq. (4)]. The reduction of $w$ increases the pulsating frequency $\omega$ and therefore the resis-

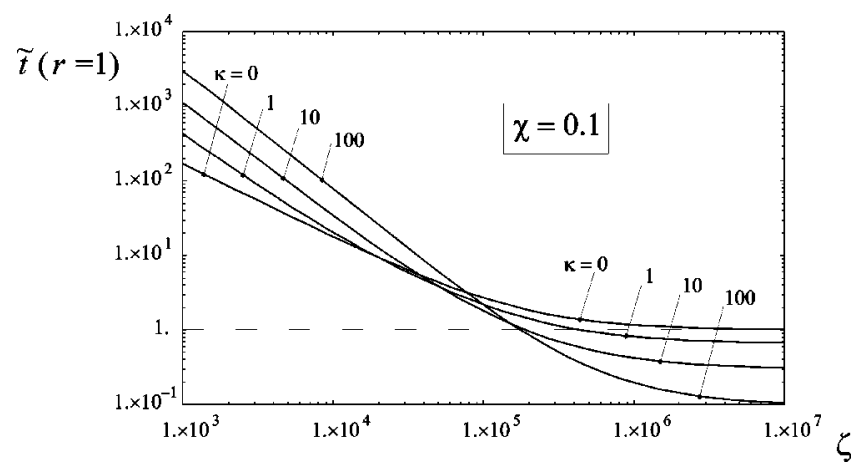

FIG. 9. The time for complete dewetting $\tilde{t}(r=1)$ as a function of the hysteretic dimensionless parameter $\zeta$ : the smaller $\zeta$ the larger the viscoelastic losses in the rubber. Results are shown for four different values $(\kappa$ $=0,1,10,100)$ of the dimensionless parameter $\kappa$.

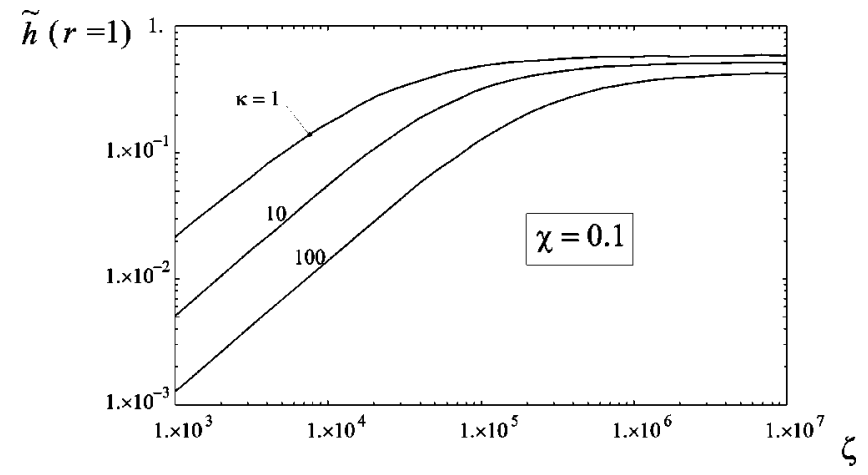

FIG. 10. The dimensionless thickness $\widetilde{h}$ of the liquid just at the end of the dewetting transition $(r=1)$ as a function of the dimensionless parameter $\zeta$ : the smaller $\zeta$ the larger the hysteretic losses into the rubber. Results are shown for three different values $(\kappa=1,10,100)$ of the dimensionless pressure parameter $\kappa$.

tant force [see Eqs. $(5,21)]$ due to the viscoelastic behavior of the rubber. This, in turn, slows down the spreading velocity of the dry patch.

It is clear that a higher pressure amplifies this effect, in that it causes a reduction of the fluid film thickness. Therefore we expect that at sufficiently higher pressures a liquid rim may remain trapped, between the rubber and the rigid wall, with almost zero spreading velocity. It should be possible to test this prediction experimentally. For instance, for tire rubber $\tau_{R} \simeq 10^{-4}-10^{-3} \mathrm{~s}$ and for water $\eta \simeq 10^{-3} \mathrm{~Pa}$ we get $\zeta \simeq 10^{3}$. Thus, in this case the limiting behavior predicted in Fig. 9 for the lowest values of $\zeta$ should be obtained. On the other hand, if the liquid is chosen to be a high viscosity fluid $\eta \simeq 10-100 \mathrm{~Pa}$ we get $\zeta \simeq 10^{6}-10^{7}$ (and even more if a PDMS rubber is chosen). In this last case the opposite limiting behavior should be observed.

Thus for viscoelastic materials the scaling relation $R$ $\simeq t^{3 / 4}$ does not always hold. For instance, a linear relation $R \propto t$ can sometimes be obtained as shown in Fig. 11 for $\kappa$ $=1, \zeta=10^{6}, \chi=0.1$. We note that $R \propto t$ has also been observed experimentally. ${ }^{20}$

The treatment presented above has been performed at the level of scaling laws, that is, numerical coefficients have been neglected. An exact analysis of the dewetting transition is, indeed, nearly impossible mainly because there is no methodology able to exactly calculate the viscous dissipation

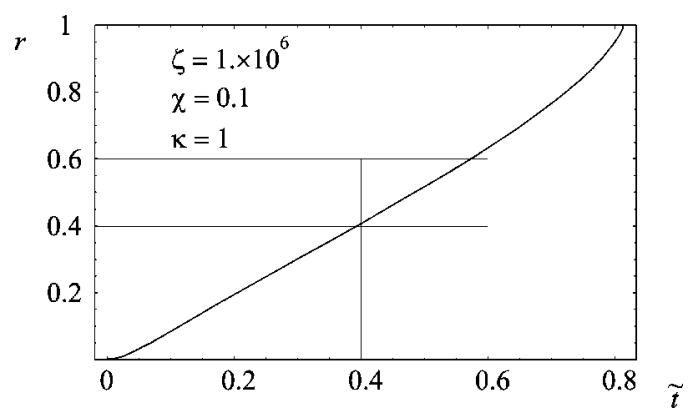

FIG. 11. The dimensionless radius $r$ of the dry patch as a function of the dimensionless time $\tilde{t}$. Results are reported for a viscoelastic material with the following values: $\kappa=1, \zeta=10^{6}, \chi=0.1$. Observe that $r$ is almost proportional to time $\tilde{t}$, as also observed in some experiments (Ref. 20). 
in the rubber and the profile of the rim. However, we expect that the basic physics of the dewetting transition is correctly described. The contribution to the energy dissipation of the small-scale viscoelastic processes close to the tip of the rim, which we have neglected, would produce an additional reduction of the growth rate of the dry zone, thus reinforcing the above depicted scenario.

\section{CONCLUSION}

We have studied the dewetting transition at soft viscoelastic interfaces, e.g., a rubber ball squeezed against a rigid flat substrate in a nonwetting liquid. It has been shown that the viscoelastic losses in the rubber may strongly slow down the dewetting, i.e., the spreading velocity of the dry patch. Moreover an unexpected behavior has been predicted: for viscoelastic materials, increasing the squeezing pressures does not necessarily increase the speed of the transition, as it may be expected, but may result in a fast reduction of the film thickness, which in turn increases the hysteretic losses in the rubber thus producing a fast reduction of the spreading velocity of the dry zone. As a consequence of this it is expected that at sufficiently high pressures a liquid rim can remain trapped, with almost zero spreading velocity, between the rubber and the substrate. The theory here proposed adopts a very simple rheological model to describe the hysteretic behavior of rubber, but the model can be extended to take into account that real rubber exhibits a wide distribution of relaxation times. Our prediction should be easy to test experimentally.

\section{ACKNOWLEDGMENT}

G.C. acknowledges the IFF, FZ-Juelich for support during one month visit where most of this research project was performed.

${ }^{1}$ A. D. Roberts and D. Tabor, Proc. R. Soc. London, Ser. A 325, 323 (1971).

${ }^{2}$ A. D. Roberts, in The Physics of Tire Friction: Theory and Experiment, edited by D. F. Hays and A. L. Browne (Plenum, New York, 1974).

${ }^{3}$ B. N. J. Persson and F. Mugele, J. Phys. Condens. Matter 16, R295 (2004).

${ }^{4}$ F. Brochard-Wyart, A. Buguin, P. Martin, A. Martin, and O. Sandre, J. Phys. C 12, A239 (2000).

${ }^{5}$ T. Becker and F. Mugele, J. Phys.: Condens. Matter 15, S321 (2003).

${ }^{6}$ F. Mugele and M. Salmeron, Phys. Rev. Lett. 84, 5796 (2000).

${ }^{7}$ B. N. J. Persson, Sliding Friction: Physical Principles and Applications, 2nd ed. (Springer, Heidelberg, 2000).

${ }^{8}$ J. N. Israelachvili, Intermolecular and Surface Forces (Academic Press, London, 1995).

${ }^{9}$ M. L. Gee, P. M. McGuiggan, and J. N. Israelachvili, J. Chem. Phys. 93, 1895 (1990).

${ }^{10}$ B. N. J. Persson and E. Tosatti, Phys. Rev. B 50, 5590 (1994).

${ }^{11}$ P. G. de Gennes, Rev. Mod. Phys. 57, 827 (1985).

${ }^{12}$ F. Brochard-Wyart and P. G. de Gennes, J. Phys. C 6, A9 (1994).

${ }^{13}$ P. Martin and F. Brochard-Wyart, Phys. Rev. Lett. 80, 3296 (1998).

${ }^{14}$ A. Martin, A. Buguin, and F. Brochard-Wyart, Langmuir 17, 6553 (2001).

${ }^{15}$ B. N. J. Persson, A. I. Volokitin, and E. Tosatti, Eur. Phys. J. E 11, 409 (2003).

${ }^{16}$ F. Brochard-Wyart, J. Phys. II 4, 1727 (1994).

${ }^{17}$ F. Brochard-Wyart and P. Martin, J. Adhes. 67, 139 (1998).

${ }^{18}$ P. G. de Gennes, Langmuir 12, 4497 (1996).

${ }^{19}$ P. Martin, P. Silberzanm, and F. Brochard-Wyart, Langmuir 13, 4910 (1997).

${ }^{20}$ E. Verneuil, J. Clain, A. Buguin, and F. Brochard-Wyart, Eur. Phys. J. E 10, 345 (2003). 